\title{
Non-Modified Ultrasound-Responsive Gas Vesicles from Microcystis with Targeted Tumor Accumulation
}

\author{
Huan Long ${ }^{1,2, *}$ \\ Xiaojuan Qin $^{3, *}$ \\ Rui $X u^{1,2}$ \\ Chunlei $\mathrm{Mei}^{4}$ \\ Zhiyong Xiong ${ }^{5}$ \\ Xuan Deng' \\ Kaiyao Huang ${ }^{1,2}$ \\ Huageng Liang $\mathbb{D}^{5}$ \\ 'Key Laboratory of Algal Biology, \\ Institute of Hydrobiology, Chinese \\ Academy of Sciences, Wuhan, Hubei \\ Province, 430072, People's Republic of \\ China; ${ }^{2}$ University of Chinese Academy \\ of Sciences, Beijing, 100049, People's \\ Republic of China; ${ }^{3}$ Department of \\ Ultrasound, Union Hospital, Tongji \\ Medical College, Huazhong University of \\ Science and Technology, Wuhan, 430022, \\ People's Republic of China; ${ }^{4}$ Tongji \\ Medical College, Huazhong University of \\ Science and Technology, Wuhan, 430022, \\ People's Republic of China; ${ }^{5}$ Department \\ of Urology, Union Hospital, Tongji \\ Medical College, Huazhong University of \\ Science and Technology, Wuhan, 430022, \\ People's Republic of China
}

*These authors contributed equally to this work

Correspondence: Kaiyao Huang; Huageng Liang

Email leonard19800318@hust.edu.cn;

huangky@ihb.ac.cn

\begin{abstract}
Introduction: Ultrasonic molecular imaging (UMI) technology has attracted increasing interest because of its low cost and capability to evaluate changes rapidly and noninvasively at the cellular and molecular levels. The key material of this technology is ultrasound-responsive gas vesicles (GVs). GVs synthesized by conventional chemical methods have several limitations, such as high costs, low yields, and complex production processes. In comparison, biosynthesized GVs have the advantages of high stability, a low risk of toxicity, genetic engineering characterization, easy post modification and drug loading potential. However, translational studies of their biosynthesis are still in their infancy; in particular, the duration of GVs in the circulatory system is essential for the usage of UMI in biomedicine and the clinic.
\end{abstract}

Results: Here, we report novel GVs biosynthesized by the cyanobacterium Microcystis, which have a moderate size, a negative zeta potential, a rod-like morphology, and a proteinshelled gas-contained structure. These GVs without any chemical modifications could be detected in the mice circulatory system for more than 10 hours by clinically used ultrasound scanners. In particular, GVs can accumulate in tumors via the enhanced permeation and retention (EPR) effect 11 hours post-injection, and lasting at least 2 hours, which might be a potential aid for tumor diagnosis. Furthermore, pathological and hematological study suggested that GVs are safe for the host.

Conclusion: We concluded that the GVs synthesized by Microcystis without any modifications have UMI potential for systemic evaluation as well as tumoral diagnosis after intravenous injection.

Keywords: ultrasonic molecular imaging, gas vesicles, biosynthesis

\section{Introduction}

Ultrasonic molecular imaging technology has attracted increasing interest due to its cost, portability, and capability to evaluate changes rapidly and noninvasively at the cellular and molecular levels. Ultrasound-sensitive gas vesicles (GVs), as ultrasonic molecular imaging probes, play a key role in this technology, functionalized to have both ligand loading and contrast imaging properties.

Currently, ultrasound-sensitive gas vesicle synthesis has focused on chemical synthetic methods. Some commercially available gas vesicles, such as SonoVue, Sonovist and Levovist, are efficaciously used in the diagnosis of tumors, cardiovascular malformations, and injuries to some organs. ${ }^{1-3}$ However, chemically synthesized GVs have limited widespread applications in the clinic due to their high cost, low yield, complex synthesis, and potential toxicity risks.

In recent years, some biosynthetic GVs extracted from bacteria or algae have been developed as novel ultrasound-sensitive bubbles, exhibiting promising 
advantages. Shapiro et al first reported that protein-shelled, gas-filled GVs from Anabaena flos-aquae (Ana) and Halobacterium NRC-1 (Halo) with widths of 45-250 nm, lengths of 100-600 nm, with cylindrical and biconical shapes respectively, provided stable ultrasound contrast. ${ }^{4}$ In addition, they fabricated Escherichia coli containing novel genetically engineered GVs to ultrasonically image the colon after local injection, opening up an opportunity for GV use in biomedicine. ${ }^{5}$

The challenges may arise from screening GVs with suitable properties including the surface charge, shape, size of nanoparticles to reduce the enormous influences of the amounts of cells and biomolecules in the blood circulation. ${ }^{6}$ Especially, with enough time of circulation in the bloodstream, some nanoparticles may pass through the defective tumor vessels to accumulate in tumor tissue, posing potential of targeted imaging and drug release. ${ }^{7}$ The PEGylation remains the gold-standard of nanoparticle modifications to stably circulate in bloodstream, which produce a hydration layer to reduce the impact of proteins and cellular constituents. ${ }^{8}$ To explore the usage of intravenous injection, the GVs were also PEGylated, sustained over $48 \mathrm{~h}$ in the bloodstream. ${ }^{9}$ However, nanomedicine PEGylation induced adverse effects have been increasingly causing attention recently, which included rapid clearance of nanomedicine, leading to loss of efficacy, or severe hypersensitivity reaction, sometimes causing anaphylactoid shock with death. ${ }^{10-12}$ Native GVs without any modification may reduce the severe side effect from PEGylation. A recent study reported that native GVs extracted from Halobacteria were almost disappeared from the bloodstream 120 min after intravenous injection, ${ }^{13}$ which is far from the meeting the needs of functional or pathologic evaluations of different organs. So far, the long-term existence of biosynthetic gas vesicles in circulation has not been reported.

Among the kinds of cyanobacteria, Microcystis sp. (Cyanophyta, Cyanophyceae, Chroococcales) is a genus of globally widespread single-cell cyanobacteria that is a potential biological resource for the biosynthesis of ultrasound contrast agents because of its enrichment of GVs, simple culture method, low cost of GV extraction and high GV stability. ${ }^{14,15}$ Herein, we studied the characterization GVs from Microcystis, and ultrasonically demonstrated their circulation time and tumor accumulation. We confirmed that biosynthetic GVs from Microcystis could sustain a long period of blood circulation, presenting great potential for systemic imaging, and they could further accumulate in tumors, providing the potential for ultrasonically targeted diagnosis.

\section{Materials and Methods}

Transmission Electron Microscopy (TEM) and Negative-Staining Electron

\section{Microscopy}

The electron microscopic experiments in this study were carried out on the platform of the Institute of Hydrobiology, Chinese Academy of Sciences. TEM experiments: The algae cells or tumor tissues were fixed at room temperature for $2-4$ hours with $2.5 \%$ glutaraldehyde $(\mathrm{pH}=$ 7.0), washed with 0.1 M PBS 3 times (15 minutes per wash), fixed with $1 \%$ osmium acid $(\mathrm{pH}=7.0)$ for 2.5 hours and then washed with PBS 3 times. Dehydration: Cells were dehydrated with $30 \%, 50 \%, 70 \%, 80 \%, 90 \%, 100 \%$, and $100 \%$ ethanol for 10 minutes each time, then 100\% ethanol:100\% acetone $(1: 3 ; 1: 1 ; 3: 1)$ for 10 minutes each time, and finally, with $100 \%$ acetone twice for 10 minutes each time. Embedding: epoxy resin (Spon 812, GS02660) was used as the embedding agent in this experiment. The dehydrated samples were permeated step by step as follows: in a 2:1 acetone/embedding agent for 30 minutes, in a 1:1 acetone/ embedding agent for 1 hour, in a 1:2 acetone/embedding agent overnight, and in pure embedding agent for 1 hour; the permeated samples were transferred into capsules for embedding, aggregated at $37^{\circ} \mathrm{C}$ for 12 hours, and then the temperature was increased to $60^{\circ} \mathrm{C}$ for 48 hours. Slices: Slices were made with a Leica U7 ultrathin slicing machine. The thickness of the slices was $70 \mathrm{~nm}$. Double staining: The slices were double stained with $2 \%$ uranium acetate and $3 \%$ lead citrate for 5-8 minutes. Observation: A Hitachi 7700 microscope was used for electron microscopy observations.

Negative staining electron microscopy: $5 \mu \mathrm{L}$ of sample was dropped onto a copper mesh with carbon support film. The copper mesh was held with tweezers for 3-5 minutes, and then the excess liquid from the edge of the copper mesh was absorbed using filter paper. A 3\% phosphotungstic acid negative staining solution was pipetted onto the sample. The sample was stained for 3-5 minutes, and then the staining solution was removed with filter paper. The sample was dried for transmission electron microscopy observation (Hitachi 7700).

\section{Extraction of Gas Vesicles}

The isolation process of GVs from $300 \mathrm{~mL}$ of logarithmicgrowing Microcystis (OD500 $=0.6-1.0)$ is described here. First, $300 \mu \mathrm{L}$ of penicillin $\mathrm{G}$ (stored concentration: $0.16 \mathrm{mg} /$ $\mathrm{mL}$; final concentration: $0.16 \mathrm{mg} / \mathrm{L}$ ) and $300 \mu \mathrm{L}$ of $\mathrm{MgCl}_{2}$ (stored concentration: $1 \mathrm{mmol} / \mathrm{mL}$; final concentration: 1 
$\mathrm{mmol} / \mathrm{L}$ ) were added to the cultured Microcystis on the bench. The culture was placed in a light incubator at a slow speed (70 rotations/min) for 5 hours. Cell collection: The algal cells were centrifuged at $4{ }^{\circ} \mathrm{C}$ and $600 \mathrm{~g}$ for 15 minutes and suspended in fresh BG11 medium. Lysis: Lysozyme at a final concentration of $0.1 \mathrm{mg} / \mathrm{mL}$ was added to the cell suspension and incubated at $37^{\circ} \mathrm{C}$ for 1 hour. Osmotic impact: Glycerol (final concentration: $1 \mathrm{~mol} / \mathrm{L}$ ) was added to the pyrolysis products and mixed well. Then, a 3-fold volume of $40 \mathrm{mmol} / \mathrm{L}$ Tris- $\mathrm{HCl}(\mathrm{pH}=7.7)$ was quickly added to percolate the cells. The mixture was placed in a $4^{\circ} \mathrm{C}$ refrigerator overnight. Collection of the GVs: The algal cell suspension was centrifuged after decomposition for 3 hours at $4^{\circ} \mathrm{C}$ and $400 \mathrm{~g}$. The white GV layer with a meniscus shape on the surface after centrifugation was collected by syringe. The GVs were washed with PBS and centrifuged at $4{ }^{\circ} \mathrm{C}$ and $400 \mathrm{~g}$ for 3 hours. The collection and washing steps were repeated. The washed GVs were transferred to a small spiral bottle and stored at $4^{\circ} \mathrm{C}$.

\section{In vitro Ultrasound Imaging}

Imaging phantoms were prepared from $1 \%$ agarose in pure water. Microcystis cells or GV samples with gradient concentrations were mixed 1:1 with melted $1 \%$ agarose, and $200 \mu \mathrm{L}$ of the mixture was quickly loaded into phantom wells. Imaging was performed using a clinical ultrasound machine (GE Medical Systems, Italy GE LOGIQ E9) and a US probe (L9, transmit frequency: $8.4 \mathrm{MHz}$ ). The ultrasonic probe was placed directly on the surface of the agarose dish with $100 \%$ maximum output power mode (MI: 1.2) to perform ultrasonic harmonic imaging.

\section{Injection of GVs into the Caudal Vein of Mice and Ultrasonic Imaging of the Liver}

Healthy nude mice were anesthetized with $10 \%$ chloral hydrate at a dose of $30 \mu \mathrm{L} / 10 \mathrm{~g}$. The anesthetic method was abdominal subcutaneous anesthesia. After anesthesia, $300 \mu \mathrm{L}$ of gas vesicles (OD500=2-3) or PBS (control group) was injected into the caudal vein of the 18 nude mice with an insulin syringe. 2 mice for each time point, as no enhanced signal can be detected after the GV is dispersed by ultrasonic pressure. Ultrasonic imaging of the liver was performed with a $9 \mathrm{~L}$ probe $0,1,2,4,5,7$, 8, 10, and 11 hours after injection (GE Medical Systems, Italy GE LOGIQ E9). $60 \%$ output power mode (MI: 0.8 ) was used to perform ultrasonic imaging.

\section{Establishment of Bladder Cancer Model Mice}

Cell culture: A bladder cancer cell line (EJ cells, commercially purchased from the China Center for Type Culture Collection) was cultured in RPMI 1640 plus $10 \%$ FBS medium at $37^{\circ} \mathrm{C}$ and with $5 \% \mathrm{CO}_{2}$. Collection of EJ cells: Cells in the logarithmic growth phase were digested with $0.25 \%$ trypsin for $3-5$ minutes, and then the suspension was centrifuged at $800 \mathrm{~g}$ for 5 minutes. The cell pellet was washed with PBS once, suspended in RPMI 1640 medium and put on ice. The collected cells were subcutaneously inoculated into nude mice within 30 minutes. Tumor implantation: BALB/c nude mice 4-6 weeks old were selected for the present study. The bladder cancer tumor model was established by subcutaneous injection of EJ cells. Briefly, a $100 \mu \mathrm{L}$ cell suspension with a concentration of $3-4 \times 10^{7}$ cells $/$ $\mathrm{mL}$ was inoculated under the axilla of each nude mouse. The skin of each nude mouse was disinfected with $75 \%$ ethanol before injection. The formation of an oval skin mound after injection indicated that the inoculation was complete. Obvious tumorigenesis was observed approximately 10-14 days after inoculation. The nude mice were used when the subcutaneous tumors of the mice were approximately $0.8-1.2 \mathrm{~cm}$ in diameter. All experimental protocols involving animals were approved by the Institutional Animal Care and Use Committee at Tongji Medical College, Huazhong University of Science and Technology (IACUC Number: 2119). The animal study was carried out in compliance with the guidance suggestion of Hubei Provincial Experimental Animal Research Centre (Certificate number: TY20160614).

\section{Ultrasound Imaging of Tumors at Different Time Points After the in situ Injection of GVs}

To evaluate the ultrasound-enhanced ability of GVs in tumors, BALB/c mice bearing EJ cell line tumors were slowly injected with $150 \mu \mathrm{L}$ of gas vesicles (OD500=1-2) into the tumors. Ultrasound imaging was performed at 0 minutes and 15 minutes after injection. Ultrasound imaging using $100 \%$ output power mode (MI: 1.2 ) was performed at 0 minutes and 15 minutes after injection.

\section{Ultrasound Imaging of Tumors at Different Time Points After Intravenous Injection of GVs}

Several subcutaneous bladder tumor model mice were injected with $300 \mu \mathrm{L}$ of gas vesicles (OD500=2-3) into 
the caudal vein with an insulin syringe. Ultrasound imaging of the liver was performed $0,1,2,4,5,7,8,10,11$, 13 and 24 hours after injection. Ultrasound imaging of tumors was performed 11, 13, 15 and 24 hours postinjection.

\section{Immunofluorescence Staining}

Three subcutaneous bladder tumor model mice were injected with $300 \mu \mathrm{L}$ of gas vesicles (OD500 between 2 and 3 ) into the caudal vein with an insulin syringe as the experimental group. The other two mice were injected with $300 \mu \mathrm{L}$ of PBS as the control group. Thirteen hours after injection, cervical dislocation was performed. After dissection, tumor samples from both groups were collected and fixed with $4 \%$ paraformaldehyde. These samples were then sent to Wuhan Servicebio Technology Company for paraffin-embedded sectioning and immunofluorescence staining. The polyclonal antibody of Microcystin GvpC (working dilution: 1/100) was used for immunostaining. The nucleus was stained with DAPI. The slices were scanned with a Pannoramic MIDI (3D HISTECH).

\section{Biosafety Testing After Gas Vesicle Injection in vivo}

Four 2-month-old nude mice were selected for histological experiment. Two were injected with $300 \mu \mathrm{L}$ of GVs $(\mathrm{OD} 500=2-3)$ into the caudal vein, and the other two were injected with $300 \mu \mathrm{L}$ of PBS as the control group. Survival was observed for 4 weeks, and then cervical dislocation was performed. After dissection, the main organs, such as the heart, liver, spleen, lungs and kidneys, were harvested for morphological comparison and fixed with $4 \%$ paraformaldehyde for 24 hours. These samples were sent to Wuhan Servicebio Technology Company for paraffin-embedded sectioning and HE staining assays. The process of sectioning included dehydration, transparency, wax immersion, embedding, slicing, and dewaxing. Then, the nucleus was stained with hematoxylin, the cytoplasm was dyed with eosin, and finally dehydration and mounting. The HE-stained slices were observed under a light microscope (Olympus BX53, Nuance software). Twelve 2-month-old C57 mice were selected for hematological experiment. Six were injected with $300 \mu \mathrm{L}$ of $\mathrm{GVs}$ $(O D 500=2-3)$ into the caudal vein, and the other six were injected with $300 \mu \mathrm{L}$ of PBS as the control group. Cervical dislocation was performed 24 hours after injection, the whole blood from three GV-injected and three PBS- injected mice was sent for blood routine examinations; the serum from three GV-injected and three PBS-injected mice was sent for blood biochemistry examinations (Wuhan Servicebio Technology Company).

\section{Immune Response Assay}

Six 2-month-old C57 mice were selected for immune response assay. Three were injected with $300 \mu \mathrm{L}$ of GVs $(\mathrm{OD} 500=2-3)$ into the caudal vein, and the other three were injected with $300 \mu \mathrm{L}$ of PBS as the control group. 24 hours after GV-injection, representative cytokines including TNF-alpha, IL-1 beta and IL-6 in the serum were analyzed with ELISA kits (Invitrogen, 88-7324, 88$7013,88-7064)$ according to the manufacturer's instructions (Wuhan Servicebio Technology Company).

\section{Cell Viability Assay}

HEK293 cells (DMEM medium supplemented with $10 \%$ FBS, commercially purchased from the China Center for Type Culture Collection) and EJ cells were cultured in 96well cell plates $\left(1 \times 10^{4}\right.$ cells/well $)$ and treated with $10 \mu \mathrm{L}$ GVs $($ OD500 $=2)$ for 24 hours. Then, CCK-8 solution (Cell Counting Kit-8, Beyotime, Shanghai) was added to the plate and incubated for 1 hour. The absorbance was measured at $450 \mathrm{~nm}$ using a microplate reader (SpectraMax M5, Molecular Devices).

\section{Results}

\section{Ultrasound Contrast Imaging of GV-Containing Microcystis Cells}

The Microcystis strains FACHB930, FACHB1326, and FACHB2329 maintained at the Freshwater Algae Culture Collection at the Institute of Hydrobiology (FACHB) were used in this study. The cells of these strains were fixed and observed using transmission electron microscopy (TEM) (Figure 1A and B). GVs congregated together to form pseudovacuoles, which localized irregularly in Microcystis cells (Figure 1A, sFigure 1A). As shown in Figure 1B, the longitudinal shapes of the GVs were hollow cylinders with conical ends; the transverse shapes of the GVs were round, and when many GVs were grouped together, they were shaped like honeycombs.

After confirming that GVs were present in these strains, to further select the suitable strain to extract the GVs, the cells were ultrasonically imaged using a clinical ultrasonic scanner (GE, LOGIQ E9) and a 2D transducer (L9) at first. The contrast-enhanced effect was observed 
A

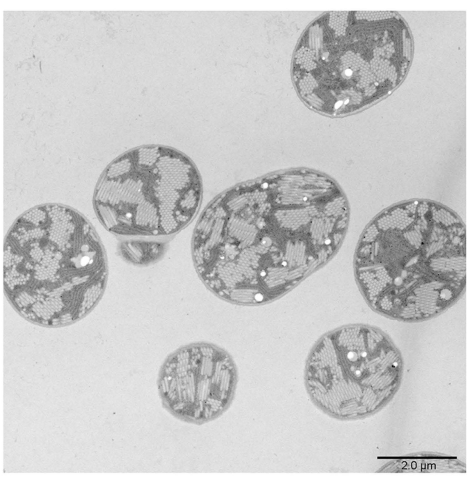

D

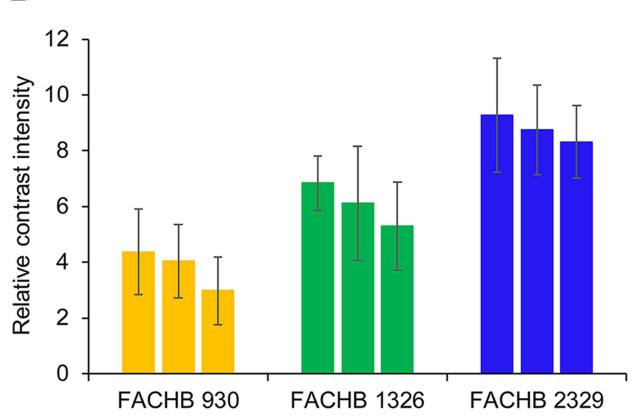

B

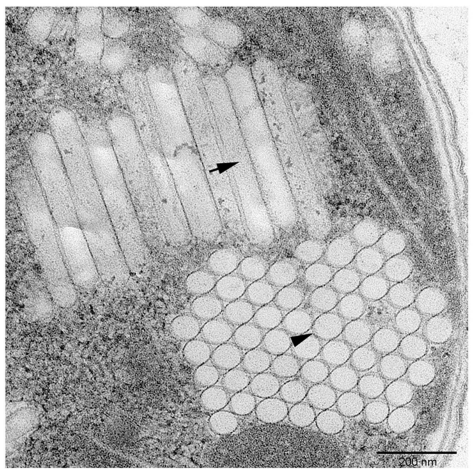

C



PBS

0.51

0.25

0.13

FACHB 1326

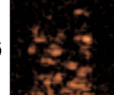

FACHB 2329

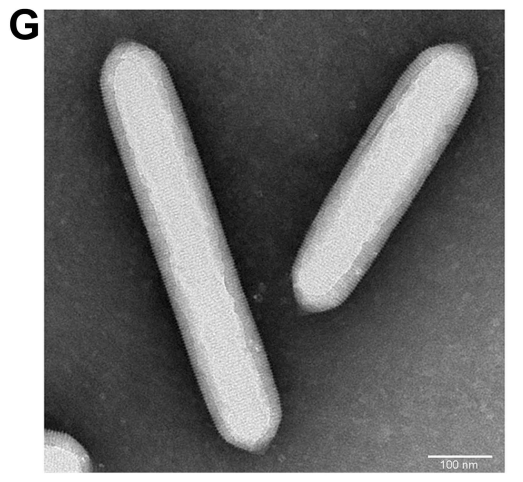

I

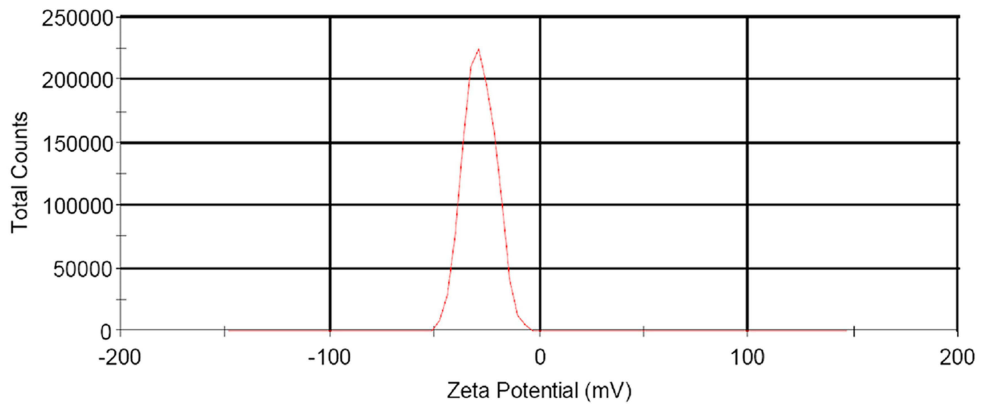

H
E

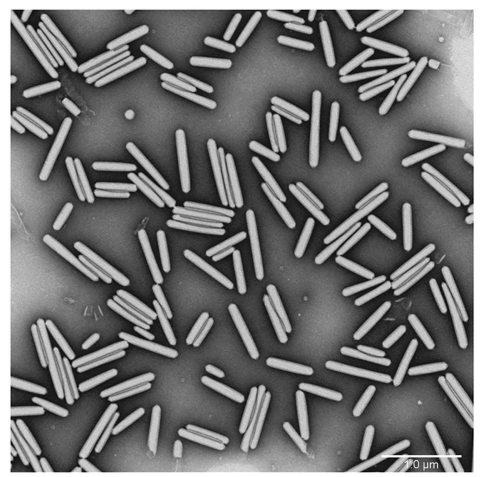

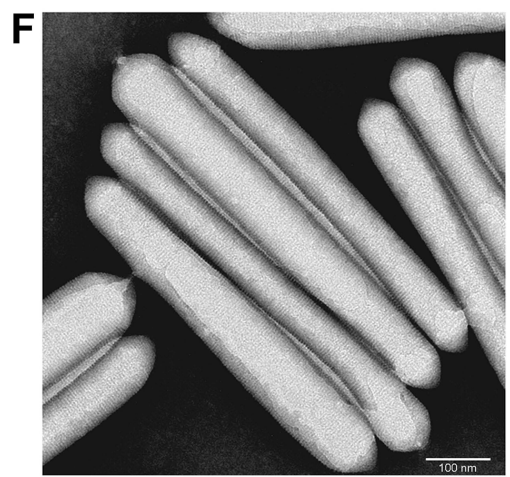


Figure I GVs can be synthesized in Microcystis, the cells and the purified GVs have enhanced contrast signal under ultrasound detection. (A and B) Transmission electron microscopy images of Microcystis cells from strain FACHB2329 at different magnifications. GVs accumulated in cells to form pseudovacuoles. The arrow indicates the longitudinal section of the GVs (B), and the arrowhead points to the transverse section of the GVs (B). (C) The cells from different Microcystis strains show concentrationdependent contrast signals under ultrasound detection. (D) Comparison of the intensity of the ultrasound contrast signal in different strains. The signal intensity was normalized by the OD500 value. (E-I) Characterization of the GVs biosynthesized in Microcystis cells. (E-G) Negative staining of isolated GVs, some of which adhered to each other $(\mathbf{F})$ and some of which were monomers $(\mathbf{G})$. (H) Size distribution analysis of the GVs. (I) Zeta potential analysis of the GVs. (J) Ultrasound imaging of PBS and GVs synthesized in Microcystis (FACHB2329) at optical density (OD500) ranging from 1.23 to 0.34 before (pre-collapse) and after (post-collapse) destructive scan. 
even with the GVs in the cells. The ultrasonic signal from the Microcystis cells could be detected at an OD500 as low as 0.13 and exhibited concentration-dependent enhancement (Figure 1C and D). Moreover, at the same concentration, the intensity of the ultrasound signal was different among the different strains. FACHB2329 displayed the best contrast effect, fitting with the TEM data that FACHB2329 cells have the highest content of GVs among these three strains (sFigure 1B). We chose FACHB2329 and its gas vesicles for follow-up study.

\section{Characterization of the GVs from Microcystis}

In order to extract the GVs from Microcystis, we optimized the method of isolating the GVs by combining the advantages of lysozyme lysis and hyperosmotic lysis, followed by centrifugally assisted flotation based on a previous study. ${ }^{16}$ By using this method, the GVs in the Microcystis cells can be separated and extracted efficiently and qualitatively. Because of the gas inside, the shape of the GVs could be clearly visualized by negative staining under an electron microscope. The GVs were hollow cylinders with conical ends, and their surface was a protein shell with a dense transverse stripe structure (Figure 1E-G). As demonstrated by TEM, the typical GV from FACHB2329 has a width of $109.3 \pm 9.9 \mathrm{~nm}$ and a length of $641.5 \pm 157.5 \mathrm{~nm}$ (sFigure $1 \mathrm{C}$ and $\underline{\mathrm{D}}$ ). In addition, the dynamic light scattering Zetasizer (Zetasizer Nano ZS90) indicated that the size of the isolated GVs ranged from 80 to $800 \mathrm{~nm}$, and the zeta potential was $-26.97 \pm 1.36 \mathrm{mV}$ (Figure 1H and I).

The negative zeta potential of the GVs were useful to reduce the protein adsorption since most proteins in bloodstream are negatively charged. ${ }^{17}$ We then evaluated the ultrasound contrast imaging of GVs in vitro. The purified GVs were placed in agarose gel phantoms with gradient dilutions for ultrasound imaging. As shown in Figure 1J, the ultrasound signal of GVs was detected at an OD as low as 0.34 and exhibited concentration-dependent ultrasoundenhanced ability in vitro. Under disruptive scanning, the GVs collapsed in the phantom.

\section{Ultrasound Imaging of Gas Vesicles in Blood Circulation}

To address whether the GVs could persist in the blood circulation, the purified GVs were injected into the caudal vein of nude mice, and then the livers were imaged by ultrasound at different time points. The liver was used to observe the circulated GVs, since most foreign particles accumulate and are cleared from there, except particles with diameters of less than $20 \mathrm{~nm}$, which were excreted from the kidney. ${ }^{18}$ As shown in Figure $2 \mathrm{~A}$, the liver showed clear contrast signal within $10 \mathrm{~s}$ after GV injection. The livers from different mice were imaged at 1, 2, 4, $5,7,8,10,11$ and 13 hours after injection, presenting a time-dependent ultrasound contrast signal (Figure 2A and $\mathrm{B})$. The most enhanced signal was obtained within $10 \mathrm{~s}$ postinjection. Afterwards, the signal decreased gradually (Figure 2B). There was an obvious contrast signal detected at 8 hours postinjection. A weak signal was still observed at 11 hours postinjection, although this signal could hardly be detected (Figure 2). Upon contrast signal appearance at various time points, the GVs were immediately smashed by the disruptive scan, indicating the ultrasound-induced drug release potential. These results confirmed that the GV synthesized in Microcystis can persist in the blood circulation system of nude mice for more than 10 hours.

\section{Ultrasound Imaging of Tumors After Intratumoral Injection}

The extracted GVs were intratumorally injected into nude mice with a tumor generated by subcutaneous injection of EJ cells. Enhanced ultrasound signals were observed in the upper part of the tumor immediately after injection, suggesting that the GVs had not yet diffused into the whole tumor tissue (Figure 3A and C). As shown in Figure 3B, 15 minutes postinjection, the whole tumor showed a relatively uniform contrast signal. Subsequently, the GVs dispersed under a disruptive scan (Figure 3B and C). These results suggested that GVs can exist in the physicochemical influences of the tumor microenvironment including high interstitial pressure and mild acid environments, which encourage the subsequent investigation of GVs accumulation in tumor after injecting intravenously.

\section{Ultrasound Imaging of Tumors After Intravenous Injection of GVs}

The GVs were injected intravenously into subcutaneous tumor-bearing nude mice via caudal vein. Then we ultrasonically imaged the tumors at 0 hours, 1 hour, 6 hours, 11 hours, 13 hours, 15 hours and 24 hours post-injection (Figure 3D-H, sFigure 2). At 11 hours post-injection, the 


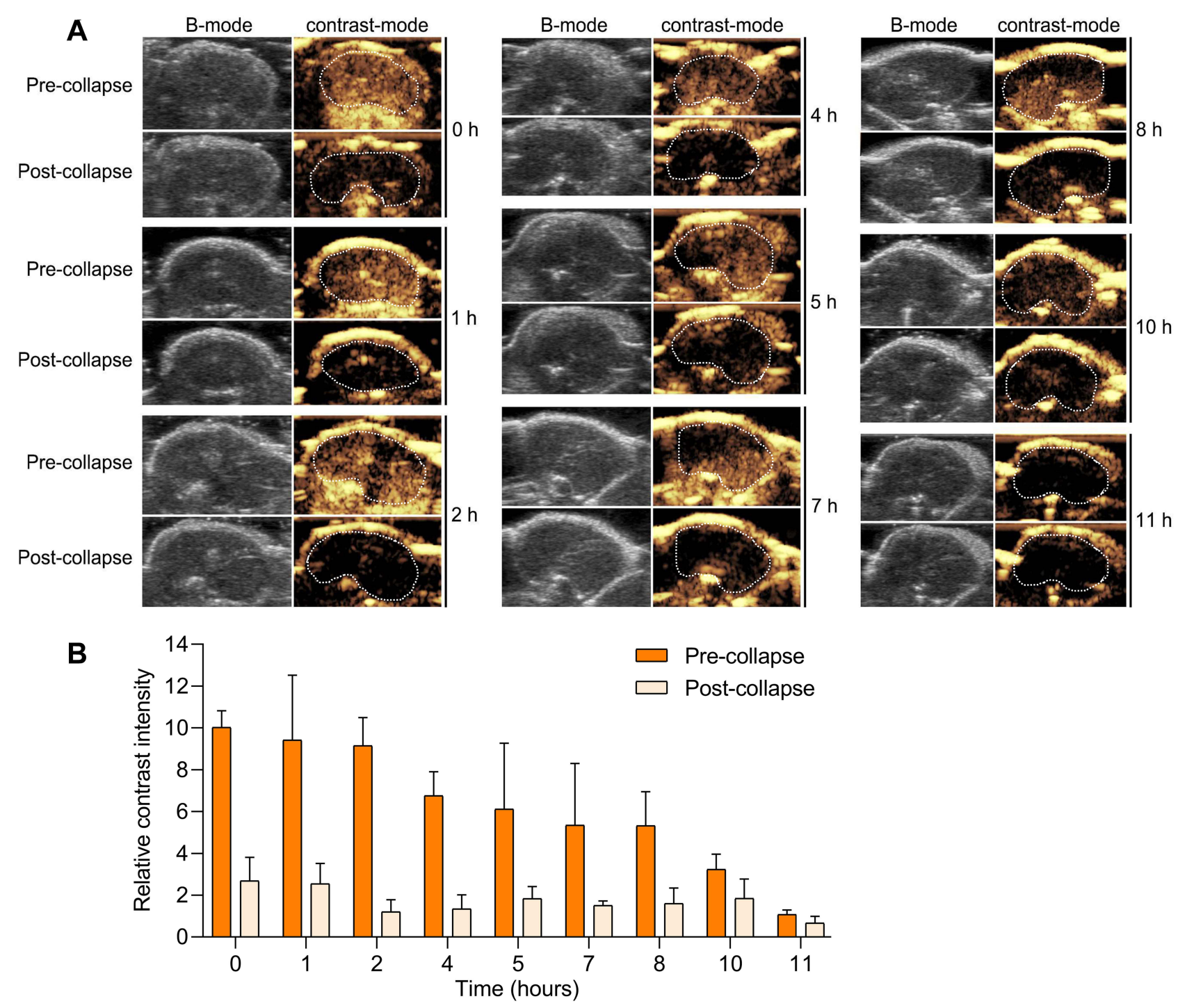

Figure 2 Ultrasound imaging of GVs synthesized in Microcystis in the livers of mice at different time points after intravenous injection. (A) The isolated GVs were injected into healthy male nude mice via the caudal vein. The contrast effect of the liver from different mice was monitored using ultrasound at 0 , I, 2, 4, 5, 7, 8, I0 and II hours. (B) Comparison of the intensity of the ultrasound contrast signal pre- or post-collapse at 0, I, 2, 4, 5, 7, 8, 10 and II hours after GV-injection.

contrast signal was detectable in the tumors (Figure 3D and $\mathrm{H}$ ), and the signal gradually increased till 13 hours post-injection (Figure $3 \mathrm{E}$ and $\mathrm{H}$ ). At 15 hours postinjection, a few gas vesicles were still detectable (Figure $3 \mathrm{~F}$ and $\mathrm{H}$ ). No enhanced ultrasound signal was detected in the tumors after 24 hours (Figure $3 \mathrm{G}$ and H), indicating that the metabolic time of the GVs in tumors was less than 24 hours.

To confirm that the GVs accumulated in tumors, the tumors were removed 13 hours after intravenous injection of GVs or PBS. The isolated tumors were fixed and stained with an antibody against gas vesicle protein $\mathrm{C}(\mathrm{GvpC})$. As shown in Figure 4A and B, the fluorescence signals were only detected in the tumor tissues when GVs were injected and not in the tumor when PBS was injected. To further confirm the accumulation of GVs in the tumor, the tumors were removed at 13 hours postinjection and fixed for TEM. Large amounts of GVs were observed within the tumor, and most of them existed in the intracellular space (Figure 4C and D; arrows). These results demonstrated that GVs could accumulate in tumors through EPR effects.

\section{Biosafety of Gas Vesicles}

To determine the biocompatibility of GVs, 2-month-old healthy BALB/c nude mice were intravenously injected with either GVs or PBS. After 4 weeks, the mice were sacrificed, and their vital organs were harvested and 

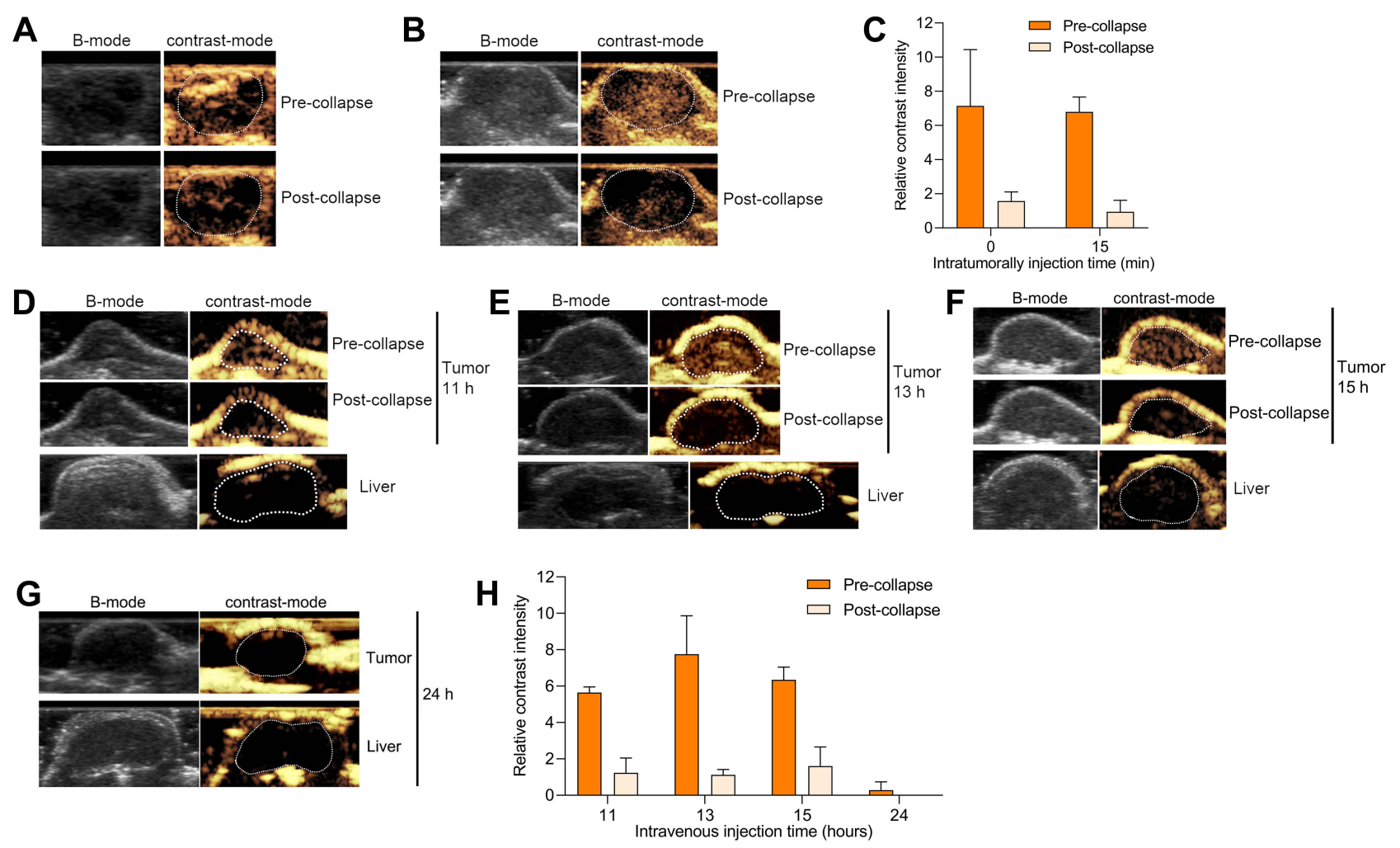

Figure 3 Ultrasound imaging of GVs in tumors. (A-C) The contrast effect of GVs after injection into tumors. (A) The contrast effect was monitored by ultrasound immediately after GVs were intratumorally injected. (B) The contrast effect was monitored by ultrasound I5 minutes after GVs were intratumorally injected. (C) Relative intensity of contrast-mode signal pre- or post-collapse in (A and B). (D-F) GVs accumulate in tumors by the EPR effect. II hours (D), I3 hours (E) and I5 hours (F) after intravenous injection of GVs, the accumulation of GVs in the tumors was detected using ultrasonic imaging. The GVs in tumors showed contrast effects and collapsed from the ultrasound mechanical force. A bare contrast-enhanced ultrasound signal could be detected in the liver. (G) Twenty-four hours after GV injection, no contrast signal was detected in either the tumor or the liver. $(\mathbf{H})$ Relative intensity of contrast-mode signal in tumors pre- or post-collapse in (D-G).

analyzed. Figure 5 shows the histological analysis of the organs, including the heart, liver, spleen, lung and kidney. There was no detectable tissue damage or other signs of impairment, such as inflammation or necrosis, in the GVinjected mice compared to the PBS-injected mice.

To further confirm the GVs' biosafety after fresh injection, the hematological data of healthy C57 mice intravenously injected with GV or PBS for 24 hours were collected and analyzed. As shown in sFigure 3A, the examined parameters of liver function including alanine aminotransferase (ALT), aspartate aminotransferase (AST), and alkaline phosphatase (ALP); the examined parameters of kidney function including blood urea nitrogen (BUN) and creatinine (CREA). AST, ALP, BUN and CREA; showed no significant differences between GVinjected groups and control groups. Only ALT in GVinjected groups was 1.3 times to that of the control groups. It might be due to the feedback effect of high protein intake in the short term, but the slight difference would not affect the liver function as the liver morphology of GV-injected groups was similar to control after 4 weeks injection (Figure 5). These results suggested that GVinjection may slightly influence the liver function in a short period, but has no affection in a long term.

For blood routine examination, the measured indexes of white blood cell (WBC), lymphocyte number (Lymph\#), monocyte number (Mon\#), granulocyte number (Gran\#), percentage of lymphocytes (Lymph\%), percentage of monocyte (Mon\%), percentage of granulocytes (Gran\%), red blood cell (RBC), hemoglobin (HGB), hematocrit (HCT), mean corpuscular volume (MCV), mean corpuscular hemoglobin $(\mathrm{MCH})$, mean corpuscular hemoglobin concentration (MCHC), and platelets (PLT) showed no statistical difference between GV-injected groups and PBS-injected groups (sFigure 3B).

In addition, the levels of TNF-alpha, IL1-beta and IL-6 in GV-injected groups were slightly higher than that of control groups, but there was no statistical difference (sFigure 4). It indicated that GVs did not induce obvious cytokine responses 24 hours after injection. The long-term effects on immune function need to be further studied. 
A

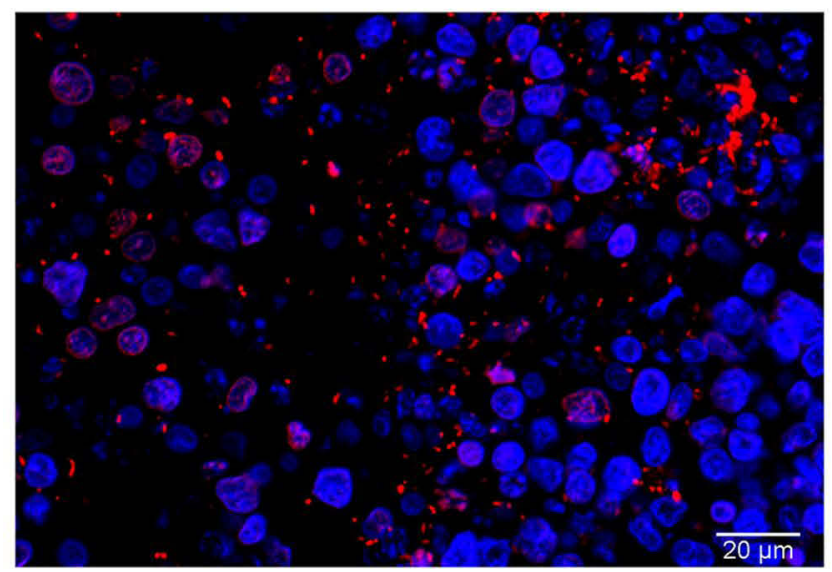

C



B



D

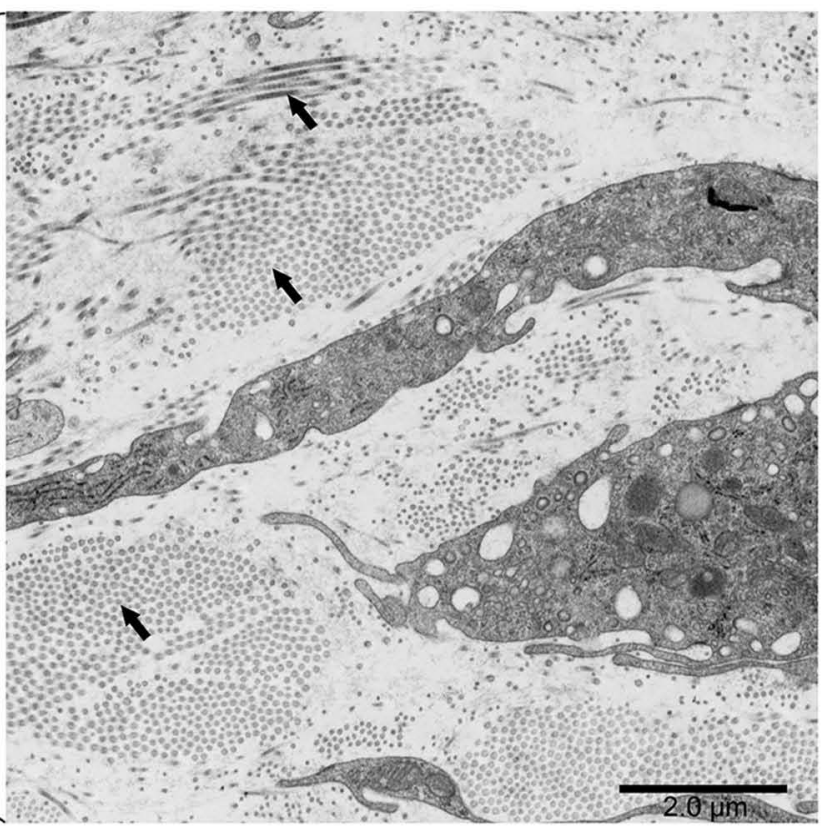

Figure $4 \mathrm{GV}$ accumulation was observed in tumors by immunostaining and transmission electron microscopy. (A) The accumulation of GVs (red dots) in the tumor 13 hours after GV injection through the caudal vein. Red signals are from immunostaining with GvpC antibody which is a marker for GVs, bule signals show nucleus that are stained by DAPI. (B) Immunostaining of the tumors from the mice in which PBS was injected. Scale bar: $20 \mu \mathrm{m}$. (C and D) The accumulation of GVs was observed in the tumor by TEM 13 hours after injection of GVs. A number of GVs were present in the intracellular space (arrows in (D)).

To verify whether GV treatment leads to harmful effect in the cellular level, we assessed the toxicity in vitro by using cell counting kit (CCK-8) assay. HEK293 and EJ cells were incubated with GVs for 24 hours respectively; no obvious influence on cell viability was found (sFigure 5).

Together, these results manifest that the GVs from Microcystis (FACHB2329) possess excellent biosafety both in vivo and in vitro, GVs-injection or GVincubation does not pose any biological safety hazards to the host or to the cells, respectively.

\section{Discussion}

Cyanobacteria are the oldest photosynthetic autotrophic organisms on earth. Gas vesicles provide buoyancy for cells so that they can migrate vertically in water, thus attaining suitable growth conditions. Many species of cyanobacteria, such as Anabaena, Planktothrix, Cylindrospermopsis, and Aphanizomenon, contain GVs. GVs from various species of cyanobacteria differ in their properties that are responsible for the different parameters of ultrasound imaging and collapse pressure due to the 



Figure 5 Histological analysis of mice main organs. Mice were injected intravenously with either $300 \mu \mathrm{L}$ PBS (control) or gas vesicles (GV injected). Then the mice were sacrificed after 4 weeks, the main organs were collected for slicing and HE staining. Scale bar $=200 \mu \mathrm{m}$.

diverse composition of the operators encoding the GVs. ${ }^{19,20}$ However, the connection has seldom been discovered between GVs and ultrasonic molecular imaging (UMI); in particular, it is unclear whether GVs can act as systematic UMI agents, with resistance to the enormous influence from the bloodstream.

After entering the blood circulation, the nanoparticles were rapidly adsorbed by the proteins on the surface to form coronas. ${ }^{6}$ Proteomic analysis has shown the existence of up to 500 proteins from the protein corona after some nanoparticles intravenously injected. ${ }^{21}$ In addition, the protein profiles in the coronas of different nanoparticles are also distinct. ${ }^{22}$ Corona formation changes the physicochemical properties and stability of the nanoparticles, finally influencing their lifetime in the bloodstream. Zeta potentials on the surface of nanoparticles are an important factor for the corona effect. Since most proteins in blood circulation exhibit a negative potential, nanoparticles with a positive potential can be reasonably adsorbed by several proteins. Conversely, nanoparticles with a negative potential would have less of a corona effect.

The present study demonstrates the capability of Microcystis as a high-quality seed resource for the biosynthesis of nano-ultrasound contrast agents. The zeta potential on the GVs from Microcystis was similar to that of a chemically synthesized gas vesicle (SonoVue ${ }^{\circledR}$ ), which has been used for systemic evaluation in the clinic. $^{23}$ Intravenously injected GVs presented the best enhanced signals in the liver at $10 \mathrm{~s}$ post-injection, followed by a time-dependent decrease in signal, which mainly correlated with the GVs suffering from influences including protein adsorption and mononuclear phagocyte system (MPS) uptake. GVs from Microcystis can persist in the blood circulation system of nude mice for more than
10 hours, there might be three reasons: 1 ). The naturally formed protein shell makes them stable for a long time; 2). The rod-like shape helps them to avoid phagocytosis; 3 ). The negative surface charge helps them escape from protein adsorption since most proteins in blood circulation are negatively charged.

After a long journey to reach the tumor after intravenous injection, GVs accumulating in the tumor can serve as targeted ultrasound imaging probes and potential drug carriers. Most nanoparticles can hardly pass-through normal vessels with epithelial gaps to enter organs or tissues. Tumor vessels are abnormal, with epithelial gaps as large as $780 \mathrm{~nm}$, facilitating nanoparticles with a suitable size to pass. ${ }^{24}$ In addition, defective lymphocyte vessels further cause nanoparticle retention in the tumor. The screened GVs were detectable 11 hours post-injection, showing the ability to accumulate in tumors via the EPR effect. However, the accumulation was confronted with some special challenges in the tumor microenvironment, such as resistance by extracellular matrix or capture by inflammatory immunocytes, ${ }^{25}$ which needs further study.

For the application of gas vesicles, some important issues should be further addressed. For example, mutation of different amino acids in the main structural protein GvpA can produce GVs with different sizes and shapes; ${ }^{26}$ mutation of structural protein GvpC or auxiliary proteins GvpN or GvpV resulted in smaller, more spindleshaped $\mathrm{GVs}^{20,27}$ and mutation of GvpM affected the synthesis efficiency of GVs. ${ }^{28}$ Genetic engineering could enrich the types of biosynthetic ultrasound nanostructures and make the process of biosynthetic ultrasound molecular sensors more precise and controllable. On the other hand, GVs could be functionalized with peptides, proteins, or other materials, ${ }^{9,29,30}$ showing their potential as drug 
carriers. Biosynthetic GVs also showed potential as a new kind of oxygen carrier to alleviate tumor hypoxia. ${ }^{31}$ Future studies can explore the role of GVs in integrated diagnosis and therapy, such as image-guided therapy.

In summary, we reported a promising UMI agent: biosynthetic GVs extracted from Microcystis. These GVs have suitable characteristics, including a moderate size, negative zeta potential, rod-like shape, and proteinshelled gas-cored structure, facilitating long-term persistence in the bloodstream. They were ultrasonic detectable in the blood circulation system for more than 10 hours, moreover, the GVs exhibited targeted tumor accumulation via the EPR effect after a long period of blood circulation. The GVs also had excellent biocompatibility with minimal damage to major organs 4 weeks after intravenous injection. This study suggested that biosynthetic GVs acting as novel UMI agents hold great potential for enhanced ultrasound imaging, in particular, for targeted tumoral imaging.

\section{Acknowledgment}

This study was supported by the National Key R\&D Program of China (2020YFA0908800) and the National Natural Science Foundation of China (81927807). We thank Professor Xudong $\mathrm{Xu}$ for kindly providing polyclonal antibody against Microcystis GvpC. Thanks to Fang Zhou, Yuan Xiao and Zhenfei Xing from the Institute of Hydrobiology, C.A.S for helping with different microscopy experiments.

\section{Disclosure}

The authors report no conflicts of interest in this work.

\section{References}

1. Liu J, Liu X, He J, et al. Percutaneous contrast-enhanced ultrasound for localization and diagnosis of sentinel lymph node in early breas cancer. Sci Rep. 2019;9(1):13545. doi:10.1038/s41598-019-49736-3

2. Senior R, Moreo A, Gaibazzi N, et al. Comparison of sulfur hexafluoride microbubble (SonoVue)-enhanced myocardial contrast echocardiography with gated single-photon emission computed tomography for detection of significant coronary artery disease: a large European multicenter study. J Am Coll Cardiol. 2013;62 (15):1353-1361. doi:10.1016/j.jacc.2013.04.082

3. Ntoulia A, Anupindi SA, Darge K, Back SJ. Applications of contrast-enhanced ultrasound in the pediatric abdomen. Abdom Radiol. 2018;43(4):948-959. doi:10.1007/s00261-017-1315-0

4. Shapiro MG, Goodwill PW, Neogy A, et al. Biogenic gas nanostructures as ultrasonic molecular reporters. Nat Nanotechnol. 2014;9 (4):311-316. doi:10.1038/nnano.2014.32

5. Bourdeau RW, Lee-Gosselin A, Lakshmanan A, et al. Acoustic reporter genes for noninvasive imaging of microorganisms in mammalian hosts. Nature. 2018;553(7686):86. doi:10.1038/nature25021

6. Shi J, Kantoff PW, Wooster R, Farokhzad OC. Cancer nanomedicine: progress, challenges and opportunities. Nat Rev Cancer. 2017;17 (1):20-37. doi:10.1038/nrc.2016.108
7. Maeda H. Macromolecular therapeutics in cancer treatment: the EPR effect and beyond. $J$ Control Release. 2012;164(2):138-144. doi:10.1016/j.jconrel.2012.04.038

8. Suk JS, Xu Q, Kim N, Hanes J, Ensign LM. PEGylation as a strategy for improving nanoparticle-based drug and gene delivery. Adv Drug Deliv Rev. 2016;99(Pt A):28-51. doi:10.1016/j.addr.2015.09.012

9. Wang GH, Song L, Hou XD, et al. Surface-modified GVs as nanosized contrast agents for molecular ultrasound imaging of tumor. Biomaterials. 2020;236:119803. doi:10.1016/j.biomaterials.2020.119803

10. McSweeney MD, Versfeld ZC, Carpenter DM, Lai SK. Physician awareness of immune responses to polyethylene glycol-drug conjugates. Clin Transl Sci. 2018;11(2):162-165. doi:10.1111/ cts. 12537

11. Gabizon A, Szebeni J. Complement activation: a potential threat on the safety of Poly(ethylene glycol)-coated nanomedicines. Acs Nano. 2020;14(7):7682-7688. doi:10.1021/acsnano.0c03648

12. Kozma GT, Meszaros T, Vashegyi I, et al. Pseudo-anaphylaxis to polyethylene glycol (PEG)-coated liposomes: roles of Anti-PEG IgM and complement activation in a porcine model of human infusion reactions. Acs Nano. 2019;13(8):9315-9324. doi:10.1021/acsnano.9b03942

13. Le Floc'h J, Zlitni A, Bilton HA, et al. In vivo biodistribution of radiolabeled acoustic protein nanostructures. Mol Imaging Biol. 2018;20(2):230-239. doi:10.1007/s11307-017-1122-6

14. Buckland B, Walsby AE. Study of strength and stability of gas vesicles isolated from a blue-green alga. Arch Mikrobiol. 1971;79 (4):327. doi:10.1007/BF00424908

15. Walsby AE. Gas vesicles. Microbiol Rev. 1994;58(1):94-144. doi:10.1128/mr.58.1.94-144.1994

16. Lakshmanan A, Lu GJ, Farhadi A, et al. Preparation of biogenic gas vesicle nanostructures for use as contrast agents for ultrasound and MRI. Nat Protoc. 2017;12(10):2050-2080. doi:10.1038/ nprot.2017.081

17. Du JZ, Mao CQ, Yuan YY, Yang XZ, Wang J. Tumor extracellular acidity-activated nanoparticles as drug delivery systems for enhanced cancer therapy. Biotechnol Adv. 2014;32(4):789-803. doi:10.1016/j. biotechadv.2013.08.002

18. Shao JD, Xie HH, Huang $\mathrm{H}$, et al. Biodegradable black phosphorus-based nanospheres for in vivo photothermal cancer therapy. Nat Commun. 2016;7. doi:10.1038/ncomms12967

19. Pfeifer F. Distribution, formation and regulation of gas vesicles. Nat Rev Microbiol. 2012;10(10):705-715. doi:10.1038/nrmicro2834

20. Tashiro Y, Monson RE, Ramsay JP, Salmond GPC. Molecular genetic and physical analysis of gas vesicles in buoyant enterobacteria. Environ Microbiol. 2016;18(4):1264-1276. doi:10.1111/14622920.13203

21. Shannahan JH, Brown JM, Chen R, et al. Comparison of nanotube-protein corona composition in cell culture media. Small. 2013;9(12):2171-2181. doi:10.1002/smll.201202243

22. Walkey CD, Olsen JB, Song FY, et al. Protein corona fingerprinting predicts the cellular interaction of gold and silver nanoparticles. Acs Nano. 2014;8(3):2439-2455. doi:10.1021/nn406018q

23. Yang H, Cai W, Xu L, et al. Nanobubble-affibody: novel ultrasound contrast agents for targeted molecular ultrasound imaging of tumor. Biomaterials. 2015;37:279-288. doi:10.1016/j. biomaterials.2014.10.013

24. Hobbs SK, Monsky WL, Yuan F, et al. Regulation of transport pathways in tumor vessels: role of tumor type and microenvironment. Proc Natl Acad Sci U S A. 1998;95(8):4607-4612. doi:10.1073/ pnas.95.8.4607

25. Zahn LM. Effects of the tumor microenvironment. Science. 2017;355 (6332):1386-1388. doi:10.1126/science.355.6332.1386-1

26. Knitsch R, Schneefeld M, Weitzel K, Pfeifer F. Mutations in the major gas vesicle protein GvpA and impacts on gas vesicle formation in Haloferax volcanii. Mol Microbiol. 2017;106(4):530-542. doi:10.1111/mmi.13833 
27. DasSarma S, Karan R, DasSarma P, Barnes S, Ekulona F, Smith B. An improved genetic system for bioengineering buoyant gas vesicle nanoparticles from Haloarchaea. BMC Biotechnol. 2013;13. doi:10.1186/1472-6750-13-112

28. Tavlaridou S, Winter K, Pfeifer F. The accessory gas vesicle protein GvpM of haloarchaea and its interaction partners during gas vesicle formation. Extremophiles. 2014;18(4):693-706. doi:10.1007/s00792014-0650-0
29. Lakshmanan A, Farhadi A, Nety SP, et al. Molecular engineering of acoustic protein nanostructures. Acs Nano. 2016;10(8):7314-7322. doi:10.1021/acsnano.6b03364

30. Shapiro MG, Ramirez RM, Sperling LJ, et al. Genetically encoded reporters for hyperpolarized xenon magnetic resonance imaging. Nat Chem. 2014;6(7):630-635. doi:10.1038/nchem.1934

31. Song L, Wang GH, Hou XD, et al. Biogenic nanobubbles for effective oxygen delivery and enhanced photodynamic therapy of cancer. Acta Biomater. 2020;108:313-325. doi:10.1016/j. actbio. 2020.03 .034

\section{Publish your work in this journal}

The International Journal of Nanomedicine is an international, peerreviewed journal focusing on the application of nanotechnology in diagnostics, therapeutics, and drug delivery systems throughout the biomedical field. This journal is indexed on PubMed Central, MedLine, CAS, SciSearch ${ }^{\mathbb{R}}$, Current Contents ${ }^{\mathbb{R}} /$ Clinical Medicine, $^{-}$
Journal Citation Reports/Science Edition, EMBase, Scopus and the Elsevier Bibliographic databases. The manuscript management system is completely online and includes a very quick and fair peer-review system, which is all easy to use. Visit http://www.dovepress.com/ testimonials.php to read real quotes from published authors. 